\title{
CONSTRUCTION OF AGRICULTURAL PRODUCTS LOGISTICS INFORMATION SYSTEM BASED ON .NET AND WAP
}

\author{
Yan Zhang ${ }^{1, *}$, Yong Liang ${ }^{1,2}$, Chengming Zhang ${ }^{1}$, Qiulan $\mathrm{Wu}^{1}$, Pingjiu $\mathrm{Ge}^{1}$ \\ ${ }^{1}$ College of Information and Engineering, Shandong Agriculture University, Taian, China, \\ 271018 \\ ${ }^{2}$ Chinese Academy of Surveying \& Mapping, Beijing, China, 100039 \\ * Corresponding author, Address: College of Information and Engineering, Shandong \\ Agriculture University, 61 Daizong Street of Taian, Shandong, 271018, P. R. China, \\ Tel: +86-538-8242497, Fax: +86-538-8249275, Email: zhangyandxy@sdau.edu.cn
}

Abstract: $\quad$ Functions and construction of agricultural products logistics system based on .NET and WAP technology are introduced in detail. The problems encountered during the process of system development and corresponding solutions are also illustrated. The Windows 2003 Server and SQL Server 2005 serve as the platform and background database server respectively, and windows are designed using the ASP.NET mobile controls. This system will be beneficial to the circulation of agricultural products in undeveloped area. The information can be released and browsed through WAP mobile phone anytime and anywhere, so a convenient means of exchanging information is provided by this system.

Keywords: $\quad$ WAP, .NET, Agricultural Product Logistics

\section{FOREWORDS}

Traditional circulation of agricultural products is keeping through fairs or market. This approach suits to the small areas while the circulation information within a large area often lags (Ratnasingam et al., 2006). With the 
development of Internet technology, network has been applied to all levels of society, and the exchange of information has become more timely and easily (Shu Geng et al., 2006). Computers has become very popular in urban areas, but it is not so realistic to buy a computer in the countryside where the economy is relatively undeveloped (Sun Wei et al., 2004). However, with the increasing improvement of living standard, mobile phone was not a luxury any more, and it has become a reality for farmers to have own cell phones. Meanwhile, the communication costs of mobile phone gradually reduced because the amounts of users increase violently. Both provide a possibility of access to Internet through mobile phones. At present, it has become popular to access to Internet using the WAP-enabled mobile phone with the standardization of wireless access protocol (WAP). In this way, users can visit the websites that provide WAP functions, view information and download resources by cell phone at any time. The WAP-based agricultural logistics information system is submitted according to these considerations.

\section{SYSTEM BASIS: .NET AND WAP}

\section{$2.1 \quad$.NET Technology}

.NET is a collection of applications supported by Web Services programming and is a technology used for the seamless interoperability between applications and computing equipments and the realization of Web interface, which is proved to personal and commercial users by Microsoft (Hou Yingchun et al., 2003). As a part of .NET framework, ASP.NET is a technology used to establish dynamic Web applications. ASP.NET applications can be written by any .NET-compatible languages such as Visual Basic .NET, C\# and J\#. Compared to the original Web technology, ASP.NET provides a programming model and structure, and can build flexible, secure and stable applications rapidly and easily. Web Forms can create powerful windows based on the web sites. Once the Web Forms established, the common user interface elements can be built using ASP.NET server controls and the tasks can be accomplished by programming. These controls allow the use of built-in reusable components and user-defined components to quickly build Web Form, and simplify your code. In this system, the ASP.NET mobile controls are used for pages design and websites development. 


\subsection{WAP Technology}

WAP is an open global standard for the communication among digital mobile phones, computer applications, Internet and personal digital assistants (PDA) (Ma Xiaojin et al., 2006). A wealth of information from Internet and a variety of business can be introduced into mobile phones and PDA wireless terminals through this protocol without any restriction on network types, network architectures, business of operators and terminal equipments. Thus, users can get the online information resources expressed in unified format through WAP-supported cell phone anytime and anywhere.

Different from the client/server architecture of Internet, the structure of WAP network consists of three parts, that is, WAP gateway, WAP phones and WAP content server. The network structure is shown in Figure 1.

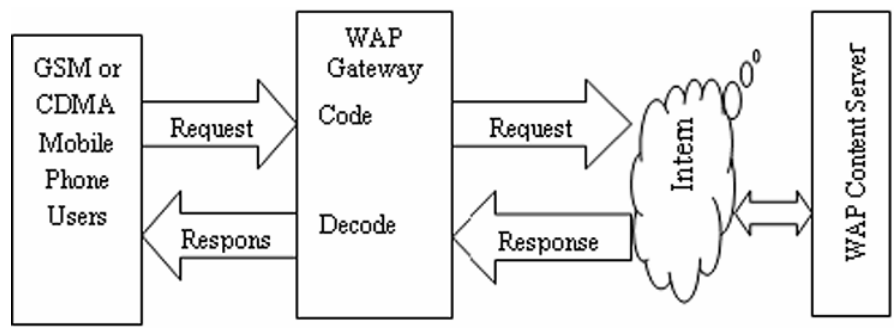

Figure 1. WAP network structure

\section{SYSTEM CONSTRUCTIONS}

\subsection{Platform Construction}

The IIS of Windows 2003 Server functions as the WAP server and SQL Server 2005 acts as the background database. Both of them construct the platform of agricultural products logistics system. To enable the server to support WAP functions and to ensure normal operation of the WAP modules, it is necessary to set up the server as follows: (1) Configured WWW services on the IIS server. (2) Add WAP-supported document types on WWW server. To achieve the transmission of WAP documents, supports of MIME that is the unique type of WAP are needed. The specific extensions and content types are shown in Table 1 (Gao Lei et al., 2003; Xu Haoyue, 2005). 
Table 1. Document types needed for WAP

\begin{tabular}{lll}
\hline Extensions & Content Types (MIME) & Remarks \\
\hline .wbmp & image/vnd.wap.wbmp & WAP-supported bit maps \\
.wml & text/vnd.wap.wml & WAP-supported WML web page text \\
& & files \\
.wmlc & application/vnd.wap.wmlc & WAP-supported WML application files \\
.wmlsc & application/vnd.wap.wmlscriptc & WAP-supported WML script application \\
& & files \\
.wmlscript & text/vnd.wap.wmlscript & WAP-supported WML script web page \\
& & text files \\
.wsc & application/vnd.wap/wmlscriptc & WAP-supported WML script application \\
& & files \\
\hline
\end{tabular}

\subsection{System Architecture}

The system structure is divided into three layers (Qi Zhiyin et al., 2004; Yang Rui et al., 2004), as is shown in Figure 2.

(1) Presentation: mainly carry out the functions of interacting with final users. This layer is some web pages and codes with extension of .aspx;

(2) Middle layer: mainly use to package business logic and rules. This layer is packaged into .NET components in applications;

(3) Data Access: interact with SQL Server Provider through the data access components in intermediate layer.

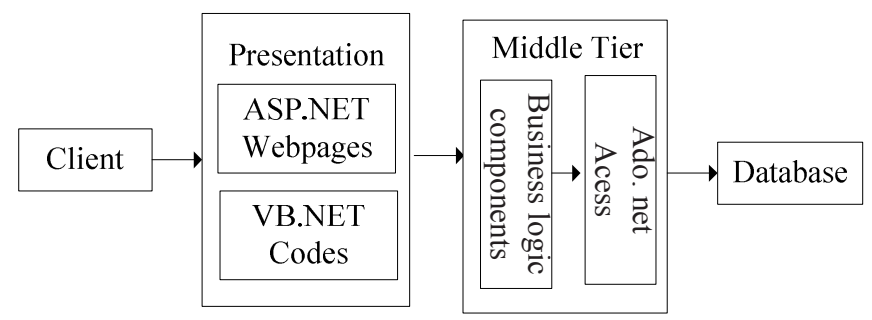

Figure 2. Architecture of system

\subsection{Functions Design}

The agricultural products logistics system contains the following modules:

(1) The latest developments: access to the agricultural products market, including the latest market news. The information is updated by the system administrator through the background database.

(2) Information Release: mainly release the information of supply and demand.

(3) Information retrieval: retrieve the information of supply and demand according to the product types entered from mobile phones. 
(4) The market price: provide the recent prices of agricultural products in different regions. Users can also retrieve the relevant prices of agricultural products according to their requirements.

The main data tables used during the system design are shown in Table 2.

Table 2. Main data tables

\begin{tabular}{llll}
\hline Fieldname & Meaning & Field name & Meaning \\
\hline InfId & ID number & Content & Content of information \\
Flag & Supply or demand & Sort & Product types \\
Price & Price & Place & Producing area \\
Time & Released time & Deadline & Valid before \\
Contact & Contact person & Phone & Phone number \\
Fax & Fax number & E-Mail & E-mail address \\
\hline
\end{tabular}

\section{PROBLEMS AND SOLUTIONS}

(1) The system needs the support of background data, and some data that used in background database management system SQL Server 2005 are imported from Microsoft Excel. When the data are imported with the DTS Wizard that is built-in SQL Server 2005, any data cannot be found in the required table. On the contrary, a table with the name of imported table plus a "\$" is generated in SQL Server, and this table has all the data imported. Although the structure of this table is similar to the original one, the data field types in this table is different from the original data and the constraints to the field length are also not the same. So, the current table is not equivalent to the original one. To import data from Excel into SQL Server 2005 correctly and to generate a table with the same name, some SQL statements are needed. The SQL statements used to import data is shown as follows:

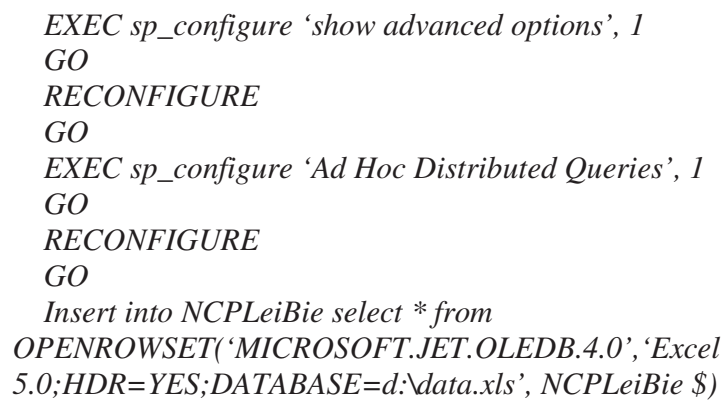

(2) In the process of dealing with information, a lot of IF statements are needed to write in the denotative layer in order to detect the information received by the clients whether accords with norms or not. Thus, the response time of mobile phones will be postponed to some extent because of 
their relatively weak capacity. The processing time of wireless terminals can be reduced by setting proper restraints for the related fields in the background database tables.

(3) In the process of writing a code to receive the agricultural products information input from the client and submit it to the background database, the information can be submitted to the Datatable. But, the background database can not be updated using the Update method of SqlDataAdapter object. For this purpose, SqlCommandBuilder object is used to create command and update data sets in data adapters automatically. The concrete realization is shown as follows:

'sqladapt agricultural products information data adapters

Dim cb As SqlCommandBuilder = New SqlCommandBuilder(sqladapt)

'tdatatable updated agricultural products data tables

Dim table As Data.DataTable = tdatatable.GetChanges

sqladapt.Update(table)

sqladapt.Fill(tdatatable)

\section{SYSTEM DEBUGGING}

In this system, both the content and the interface of WAP site are written in Chinese. Therefore, encoding command must be used to designate Chinese character sets at the beginning of programs in order that WAP browsers can display correct Chinese characters. Code is written as follows(WAP-Forum, 2002):

$<$ ? $x m l$ version $=$ "1.0" encoding $=$ " gb2312" >

The WAP browser M3Gate is adopted for system debugging. The main interface of this system is shown in Figure 3 where the menu can be selected through arrow keys of cell phones in order to access the corresponding link interface. The interface of information retrieval is shown in Figure 4 where the users can retrieve the available supply by inputting the agricultural product's name. The results of retrieval are displayed in a pattern of Figure 5. Only one item can be displayed each time and others can be seen by pressing the "next page" button. The main interface shown in Figure 3 can be returned to from arbitrary interface through the "home" button.

Completing the debugging through simulators on the PC does not mean system can be used normally. After all, there are differences between simulators with real WAP terminals. The view effect of webpage must be debugged to make some adjustment by using the real WAP mobile phones in final stage of WAP development. In addition, the standard implemented by 


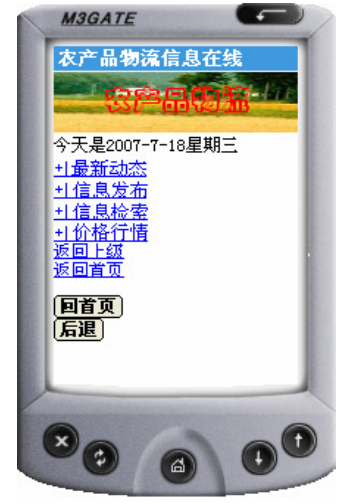

Figure 3. Main interface

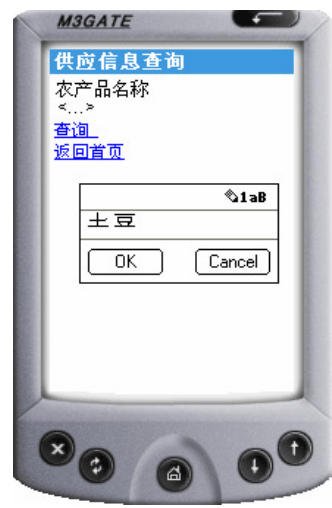

Figure 4. Supply information retrieval interface

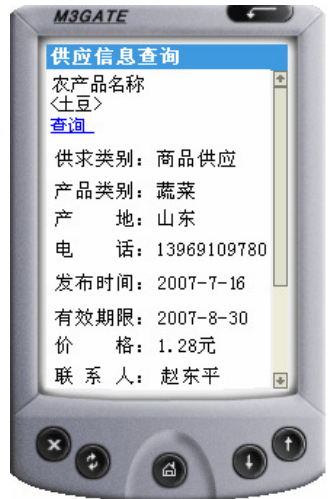

Figure 5. Retrieval results of supply information

different WAP client equipment may have differences on the details. Therefore, constant adjustments are required in the process of WAP development to make the WAP site be compatible with most equipment.

\section{CONCLUDING REMARKS}

An agricultural products logistics system is constructed by using the ASP.NET of Visual Studio 2005 as the development tools and the SQL Server 2005 as the background database. The architecture of system is given firstly. Then, the overall function modules and the main background data table structures of system are introduced by taking the actual needs of system development into account. Finally, the specific solutions in accordance with the problems encountered in the debugging process are illustrated in detail. The system is debugged and run by using the M3Gate simulator, and part operating results of the system is shown.

The agricultural products logistics information system will be beneficial to the circulation of agricultural products in economically underdeveloped regions, especially in non-urban areas. In this system that consists of WAP gateway, WAP content server and WAP terminals, users can browse agricultural products information released by others or submit his own information through WAP mobile phones anywhere and anytime. The agricultural products logistics system provides a new channel for the exchange of agricultural products information. 


\section{ACKNOWLEDGEMENTS}

The fund of this system is partly supported by Water Conservancy Science and Technology Special Funds of Shandong Province with Grant No. 200357 and the Student Research Training Plan of Shandong Agriculture University. The support is gratefully acknowledged.

\section{REFERENCES}

Gao Lei, Ren Lihong, Ding Yongsheng. Design and Implementation of WAP-based Mobile Electronic Commerce Systems. Computer Engineering and Application, Chinese, 2003, (1):215-217

Hou Yingchun, Geng Baiqiang. Application on .NET Technology in WAP. Journal of Henan Institute of Education (Natural Science), Eng., 2003, 12(3):47-49

Ma Xiaojin, Zhou Yong, Jia Shaorui, et al. Design and realization of score inquiry subsystem based on .NET and WAP mobile. Journal of Hebei Institute of Architectural Science and Technology, Chinese, 2006, 23(3):80-82

Qi Zhiyin, Li Yuchen. Building ePDMS Based on Web and WAP. Computer Engineering, Chinese, 2004, 30(7):66-68

Ratnasingam, P. The role of ecommerce adoption among small businesses: an exploratory study, Eng., International Journal of Cases on Electronic Commerce, 2006, 2(2):39-54

Shu Geng,Tian-zhi Ren, Mao-hua Wang, Technology and Infrastructure Considerations for E-Commerce in Chinese Agriculture, Eng., Agricultural Sciences in China, 2006, 6(1):1-10

Sun Wei, Wan Xiao-ning, Sun Lin-yan. Electronic commerce applied to the structure optimization in agricultural products' supply chains system. Eng., Industrial Engineering and Management, 2004, 9(5):33-41

WAP-Forum (2000a), WAP-199.WTLS (Wireless Transport Layer Security), Version 18Feb-2000 http://www.wapforum.org/what/technical.htm

$\mathrm{Xu}$ Haoyue, Wap-the foundation of the lead-in electronic commerce. Agriculture Network Information, Eng., 2005, (4):34-35

Yang Rui, Guan Xiaohong, Gao Feng, Jiang Lei, Zhao Li. Design and Implement of a Wap-based Mobile Phone Fee Query System. Eng., Microelectronics \& Computer, 2004, 21(11):92-95 